\title{
Genome Resource for Barley Leaf Stripe Pathogen Pyrenophora graminea
}

\author{
Erjing $\mathrm{Si}^{1,2}$ Yaxiong Meng, ${ }^{1,2}$ Xiaole Ma, ${ }^{1,2}$ Baochun $\mathrm{Li}^{1,3}$ Juncheng Wang, ${ }^{1,2}$ Lirong Yao, ${ }^{1,2}$ \\ Ke Yang, ${ }^{1,2}$ Yu Zhang, ${ }^{4}$ Xunwu Shang, ${ }^{1}$ and Huajun Wang ${ }^{1,2 \dagger}$ \\ ${ }^{1}$ Gansu Provincial Key Laboratory of Aridland and Crop Science/Gansu Key Laboratory of Crop \\ Improvement \& Germplasm Enhancement, Lanzhou, 730070, China \\ ${ }^{2}$ College of Agronomy, Gansu Agricultural University, Lanzhou, 730070, China \\ ${ }^{3}$ College of Life Science and Technology, Gansu Agricultural University, Lanzhou, 730070, China \\ ${ }^{4}$ Gansu Plant Seed Administrative Station, Lanzhou 730020, China
}

\begin{abstract}
Pyrenophora graminea is the causative agent of barley leaf stripe disease. In this study, the strong pathogenic isolate QWC was used to generate DNA for Illumina sequencing. After assembly, its genome size was $42.5 \mathrm{Mb}$, consisting of 264 scaffolds, and a total of 10,376 genes was predicted. This is the first genome resource available for $P$. graminea. The genome sequences of $P$. graminea will accelerate the understanding interaction of $P$. graminea and barley.
\end{abstract}

Barley leaf stripe is a seedborne disease caused by the fungus Pyrenophora graminea S. Ito \& Kurib. (anamorph Drechslera graminea (Rabenh. ex. Schltdl.) S. Ito). When the seed germinates, the mycelium of the fungus survives in the pericarp, hull, and seed coat but not in the embryo; the fungus then penetrates the coleorhiza and colonizes the plant systemically, beginning with the root tip (Platenkamp 1976). Due to the cool and humid weather conditions required for infection during germination, this disease can occur in Europe and Northern America as well as in northern Africa, Russia, India, and China. Barley leaf stripe is responsible for substantial reductions in barley yields in different cultivation areas (Zad et al. 2002). When the seed infection percentage is high, this disease results in severe yield reduction (Porta-Puglia et al. 1986). Thus far, research has been conducted regarding the genetic diversity of the pathogen (Bayraktar and Akan 2012; Si et al. 2019; Zein et al. 2010), pathogenicity differentiation (Bayraktar and Akan 2012; Si et al. 2019), resistance evaluation (Mueller et al. 2003; Tekauz 1983), resistance gene mapping (Arru et al. 2002, 2003; Biselli et al. 2010; Pecchioni et al. 1996; Tacconi et al. 2001; Thomsen et al. 1997), and candidate gene screening involved in the host-pathogen interactions (Ghannam et al. 2016); yet, little is known about the genome of $P$. graminea. In genus Pyrenophora, only three species, including $P$. teres (Ellwood et al. 2010), $P$. tritici-repentis (Moolhuijzen et al. 2018), and $P$. seminiperda (Soliai et al. 2014), have genomic information currently available. Further knowledge of the genome could be useful for understanding of the molecular basis of this disease.

The QWC isolate, collected from Yongdeng, Gansu Province in 2009, was collected for sequencing given its extensive pathogenicity compared with other isolates (Si et al. 2017). Mycelia tissue of $P$. graminea QWC isolated from barley in China was used for genomic DNA extraction with the cetyltrimethylammonium bromide method (Bayraktar and Akan 2012; Si et al. 2019). The genome size was estimated, using K-mer statistics, to be $43.9 \mathrm{Mb}$. Three genomic DNA libraries, including a 170-, 500-, and 6,000-bp library, were constructed and sequenced using an Illumina HiSeq 2000 platform. In total, 4,146 Mb of clean data were obtained by removing low-quality data, including adapters, duplications, and those reads

${ }^{\dagger}$ Corresponding author: H. Wang; whuajun@yahoo.com

The author(s) declare no conflict of interest.

Accepted for publication 18 August 2019

\section{Funding}

This work was supported by Science and Technology Innovation Funds of Gansu Agricultural University-Special funds for discipline construction (GAUXKJS-2018-082 and GAU-XKJS2018-083), Gansu Provincial Key Laboratory of Aridland Crop Science, Gansu Agricultural University (GSCS2018-6), the National Natural Science Foundation of China (CN) (31460347) Science and Technology Innovation Funds of Gansu Agricultural University-Scientific research start-up funds for openly recruited doctors (GSAU-RCZX201706); and Science and Technology Innovation Funds of Gansu Agricultural University-Sheng Tongsheng innovation funds (GSAU-STS-1735).

\section{Keywords}

barley leaf stripe, genome resource, Pyrenophora graminea 
Table 1. Summary statistics of the draft genome assembly of Pyrenophora graminea

\begin{tabular}{lr} 
Features & Numbers \\
Herbarium number & ACCC 39601 \\
Genome size $(\mathrm{Mb})$ & 43.9 \\
Total length $(\mathrm{Mb})$ & 42.5 \\
Number of scaffolds & 264 \\
Max. Scaffold length $(\mathrm{kb})$ & $3,520.8$ \\
Scaffold N $_{50}(\mathrm{~kb})^{\mathrm{a}}$ & $1,056.1$ \\
Scaffold average length $(\mathrm{kb})^{\text {GC content }(\%)}$ & 160.9 \\
Number of predicted genes & 47.4 \\
\hline
\end{tabular}

${ }^{a} \mathrm{~N}_{50}=$ scaffold length at which $50 \%$ of the total assembly length is covered.

containing $\mathrm{N}$ more than $10 \%$. A de novo genome assembly was performed with the software SOAPdenovo (Li et al. 2008, 2010).

The size of the $P$. graminea QWC genome assembly was $42.47 \mathrm{Mb}$, which is larger than that of $P$. teres (Ellwood et al. 2010). The $P$. graminea QWC genome consists of 264 scaffolds, with the scaffold length at which $50 \%$ of the total assembly length is covered of $1,056.1 \mathrm{~kb}$. The GC content is $47.4 \%$. Many of the 10,367 genes were predicted with four software programs, including GeneWise (Birney et al. 2004), SNAP (Johnson et al. 2008), Augustus (Keller et al. 2011), and Genemarkes (Ter-Hovhannisyan et al. 2008). Of the 10,367 predicted genes, 6,156 could be categorized by BLAST (Tatusov et al. 1997, 2003) to a clusters of orthologous groups (COG) category and 5,860 genes could be categorized by BLAST (Kanehisa 1997; Kanehisa et al. 2004, 2006) to a Kyoto Encyclopedia of Genes and Genomes (KEGG) category. Based on the Carbohydrate-Active Enzymes (CAZy) database (Cantarel et al. 2009), the genome contains 178 genes encoding carbohydrate esterases, 680 genes encoding glycoside hydrolases, 386 genes encoding glycosyl transferases, 15 genes encoding polysaccharide lyases, and 295 genes categorized as carbohydrate-binding modules. There were 133 secreted proteins predicted based on standards (Emanuelsson et al. 2007), of which 27 were classified as potential effectors by EffectorP 2.0 (Sperschneider et al. 2018). The summary statistics of the genome assembly are shown in Table1.

The Whole-Genome project described in this article can be accessed from NCBI SRA Bioproject ID PRJNA 544435 (https://dataview.ncbi.nlm.nih.gov/object/PRJNA544435? reviewer=3b9sgmh1cj3gs4ujgspaoiagsd). Specimens of the isolate $\mathrm{QWC}$ are available at the Agricultural Culture Collection of China (Table 1).

\section{Literature Cited}

Arru, L., Francia, E., and Pecchioni, N. 2003. Isolate-specific QTLs of resistance to leaf stripe (Pyrenophora graminea) in the 'Steptoe' $\times$ 'Morex' spring barley cross. Theor. Appl. Genet. 106:668-675.

Arru, L., Niks, R. E., Lindhout, P., Vale, G., Francia, E., and Pecchioni, N. 2002. Genomic regions determining resistance to leaf stripe (Pyrenophora graminea) in barley. Genome 45:460-466.

Bayraktar, H., and Akan, K. 2012. Genetic characterization of Pyrenophora graminea isolates and the reactions of some barley cultivars to leaf stripe disease under greenhouse conditions. Turk. J. Agric. For. 36:329-339.

Birney, E., Clamp, M., and Durbin, R. 2004. Genewise and genomewise. Genome Res. 14:988-995.

Biselli, C., Urso, S., Bernardo, L., Tondelli, A., Tacconi, G., Martino, V., Grando, S., and Vale, G. 2010. Identification and mapping of the leaf stripe resistance gene Rdg1a in Hordeum spontaneum. Theor. Appl. Genet. 120:1207-1218.

Cantarel, B. L., Coutinho, P. M., Rancurel, C., Bernard, T., Lombard, V., and Henrissat, B. 2009. The Carbohydrate-Active Enzymes database (CAZy): An expert resource for glycogenomics. Nucleic Acids Res. 37 (Database): D233-D238.

Ellwood, S. R., Liu, Z., Syme, R. A., Lai, Z., Hane, J. K., Keiper, F., Moffat, C. S., Oliver, R. P., and Friesen, T. L. 2010. A first genome assembly of the barley fungal pathogen Pyrenophora teres f. teres. Genome Biol. 11:R109.

Emanuelsson, O., Brunak, S., von Heijne, G., and Nielsen, H. 2007. Locating proteins in the cell using TargetP, SignalP and related tools. Nat. Protoc. 2: 953-971.
Ghannam, A., Alek, H., Doumani, S., Mansour, D., and Arabi, M. I. E. 2016. Deciphering the transcriptional regulation and spatiotemporal distribution of immunity response in barley to Pyrenophora graminea fungal invasion. BMC Genomics 17:256.

Johnson, A. D., Handsaker, R. E., Pulit, S. L., Nizzari, M. M., O'Donnell, C. J., and de Bakker, P. I. 2008. SNAP: A web-based tool for identification and annotation of proxy SNPs using HapMap. Bioinformatics 24:2938-2939.

Kanehisa, M. 1997. A database for post-genome analysis. Trends Genet. 13: 375-376.

Kanehisa, M., Goto, S., Hattori, M., Aoki-Kinoshita, K. F., Itoh, M., Kawashima, S., Araki, M., and Hirakawa, M. 2006. From genomics to chemical genomics: New developments in KEGG. Nucleic Acids Res. 34:D354-D357.

Kanehisa, M., Goto, S., Kawashima, S., Okuno, Y., Hattori, M. 2004. The KEGG resource for deciphering the genome. Nucleic Acids Res. 32:D277-D280.

Keller, O., Kollmar, M., Stanke, M., and Waack, S. 2011. A novel hybrid gene prediction method employing protein multiple sequence alignments. Bioinformatics 27:757-763.

Li, R. Q., Li, Y. R., Kristiansen, K., and Wang, J. 2008. SOAP: Short oligo nucleotide alignment program. Bioinformatics 24:713-714.

Li, R. Q., Zhu, H. M., Ruan, J., Qian, Q. B., Fang, X. D., Shi, Z. B., Li, Y. R., Li, S. T., Shan, G., Kristiansen, K., Li, S. G., Yang, H. M., Wang, J., and Wang, J. 2010. De novo assembly of human genomes with massively parallel short read sequencing. Genome Res. 20:265-272.

Moolhuijzen, P., See, P. T., Hane, J. K., Shi, G., Liu, Z., Oliver, R. P., and Moffat, C. S. 2018. Comparative genomics of the wheat fungal pathogen Pyrenophora 
tritici-repentis reveals chromosomal variations and genome plasticity. BMC Genomics 19:279.

Mueller, K. J., Valé, G., and Enneking, D. 2003. Selection of resistant spring barley accessions after natural infection with leaf stripe (Pyrenophora graminea) under organic farming conditions in Germany and by sandwich test. J. Plant Pathol. 85:9-14.

Pecchioni, N., Faccioli, P., Toubia-Rahme, H., Valè, G., and Terzi, V. 1996. Quantitative resistance to barley leaf stripe (Pyrenophora graminea) is dominated by one major locus. Theor. Appl. Genet. 93:97-101.

Platenkamp, R. 1976. Investigations on the infections pathway of Drechslera graminea in germinating barley. Pages 49-64 in: Royal Veterinary and Agricultural University Yearbook. Royal Veterinary and Agricultural University, Copenhagen, Denmark.

Porta-Puglia, A., Delogu, G., and Vannacci, G. 1986. Pyrenophora graminea on winter barley seed: Effect on disease incidence and yield losses. J. Phytopathol. 117:26-33.

Si, E. J., Meng, Y. X., Ma, X. L., Li, B. C., Wang, J. C., Ren, P. R., Yao, L. R., Yang, K., Zhang, Y., Shang, X. W., and Wang, H. J. 2019. Development and characterization of microsatellite markers based on whole-genome sequences and pathogenicity differentiation of Pyrenophora graminea, the causative agent of barley leaf stripe. Eur. J. Plant Pathol. 2019:1-15.

Si, E. J., Yang, S. L., Li, B. C., Ma, X. L., Wang, S. R., and Wang, H. J. 2017. Pathogenic analysis, rDNA-ITS and genetic diversity of Pyrenophora graminea in Gansu Province. J. Plant Prot. 44:84-92.

Soliai, M. M., Meyer, S. E., Udall, J. A., Elzinga, D. E., Hermansen, R. A., Bodily, P. M., Hart, A. A., and Coleman, C. E. 2014. De novo genome assembly of the fungal plant pathogen Pyrenophora semeniperda. PLoS One 9:e87045.
Sperschneider, J., Dodds, P. N., Gardiner, D. M., Singh, K. B., and Taylor, J. M. 2018 Improved prediction of fungal effector proteins from secretomes with EffectorP 2.0. Mol. Plant Pathol. 19:2094-2110.

Tacconi, G., Cattivelli, L., Faccini, N., Cchioni, N., Pecchioni, N., Stanca, A. M., and Valé, G. 2001. Identification and mapping of a new leaf stripe resistance gene in barley (Hordeum vulgare L.). Theor. Appl. Genet. 102:1286-1291.

Tatusov, R. L., Fedorova, N. D., Jackson, J. D., Jacobs, A. R., Kiryutin, B., Koonin, E. V., Krylov, D. M., Mazumder, R., Mekhedov, S. L., Nikolskaya, N. A., Rao, B. S., Smirnov, S., Sverdlov, A. V., Vasudevan, S., Wolf, Y. I., Yin, J. J., and Natale, D. A. 2003. The COG database: An updated version includes eukaryotes. BMC Bioinf. 4:41.

Tatusov, R. L., Koonin, E. V., and Lipman, D. J. 1997. A genomic perspective on protein families. Science 278:631-637.

Tekauz, A. 1983. Reaction of Canadian barley cultivars to Pyrenophora graminea, the incitant of leaf stripe. Can. J. Plant Pathol. 5:294-301.

Ter-Hovhannisyan, V., Lomsadze, A., Chernoff, Y. O., and Borodovsky, M. 2008. Gene prediction in novel fungal genomes using an ab initio algorithm with unsupervised training. Genome Res. 18:1979-1990.

Thomsen, S. B., Jensen, H. P., Jensen, J., Skou, J. P., and Jorgensen, J. H. 1997. Localization of a resistance gene and identification of sources of resistance to barley leaf stripe. Plant Breed. 116:455-459.

Zad, J., Aghakhani, M., Etebarian, R., and Okhovat, M. 2002. Barley leaf stripe disease. Meded. Rijksuniv. Gent Fac. Landbouwkd. Toegeppaste Biol. Wet. 67: 279-281.

Zein, I., Jawhar, M., and Arabi, M. I. E. 2010. Efficiency of IRAP and ITS-RFLP marker systems in accessing genetic variation of Pyrenophora graminea. Genet. Mol. Biol. 33:328-332. 